\title{
Enterococcus hirae WEHI01 isolated from a healthy Chinese infant ameliorates the symptoms of type 2 diabetes by elevating the abundance of Lactobacillales in rats
}

\author{
Min Wei, ${ }^{1}$ Enyu Gu, ${ }^{1}$ Jie Luo, ${ }^{1}$ Zhihong Zhang, ${ }^{1}$ Di Xu, ${ }^{1}$ Xueying Tao, ${ }^{1}$ Nagendra P. Shah, ${ }^{2} \odot$ and Hua Wei ${ }^{1 *}(\mathbb{C}$ \\ ${ }^{1}$ State Key Laboratory of Food Science and Technology, Nanchang University, Nanchang 330047, China \\ ${ }^{2}$ Food and Nutritional Science, School of Biological Science, University of Hong Kong, Pokfulam Road, Hong Kong, China
}

\begin{abstract}
Enterococcus hirae WEHI01 is a potential probiotic strain isolated from a healthy Chinese infant. This strain has previously been characterized as having cholesterol-lowering potential and good dairy fermentation performance. In this study, we used rat models with obesity and type 2 diabetes mellitus (T2DM) induced by a high fat and sucrose diet and low-dose streptozotocin, respectively, and we evaluated the effect of E. hirae WEHI01 on glycolipid metabolism, glycolipid-related gene expression, organ histopathology, and intestinal flora changes in the 2 models. Our results showed that administration of $5.0 \times 10^{9}$ cfu of E. hirae WEHI01 for 4 wk decreased serum lipid levels and regulated glycolipid metabolism in the liver of obese rats. Following continuous administration of the same concentration of $E$. hirae WEHI01 to a T2DM rat model for another $5 \mathrm{wk}$, E. hirae WEHI01 improved glucose tolerance, recovered body weight loss, and led to significant decreases in tumor necrosis factor- $\alpha$, IL-6, IL-10, and total bile acid in serum. We also found that E. hirae WEHI01 restored the morphology of the pancreas, kidney, and liver, and changed the composition of the gut microbiota (i.e., decreased the Shannon index, increased the Simpson index, and substantially increased the abundance of Lactobacillales). Combining the results for the obese model and the T2DM model, we speculated that beneficial effects of E. hirae WEHI01 on T2DM could be due to (1) a significant increase in $P P A R A$ expression and a tendency for increased $C Y P^{r} 7 A 1$ expression in the liver of obese rats, promoting the conversion of cholesterol into bile acid and reducing serum total bile acid levels in T2DM model rats; or (2) a change in gut microbial diversity, especially elevated Lactobacillales abundance, which reduced the total bile acid in T2DM model rats.
\end{abstract}

Received June 28, 2019

Accepted November 28, 2019.

*Corresponding author: weihua@ncu.edu.cn
These results demonstrated that E. hirae WEHI01 has the potential to ameliorate type 2 diabetes in rats and provide a promising rationale for further research into the prevention and treatment of T2DM.

Key words: type 2 diabetes, Enterococcus hirae WEHI01, gut microbiota, bile acid

\section{INTRODUCTION}

Diabetes is one of the leading causes of death around the world. The global population of people with diabetes is predicted to increase to 693 million by 2045 (Cho et al., 2018), and more than $90 \%$ of people with diabetes have type 2 diabetes mellitus (T2DM; Intagliata and Koch, 2007), which is characterized by hyperglycemia as a result of insulin resistance and relative insulin deficiency (Fujimoto, 2000). Chronic hyperglycemia can easily lead to other syndromes, such as retinopathy, nephropathy, neuropathy, and cardiovascular complications (Chen et al., 2014b). High blood glucose levels can be managed with several antidiabetic agents, including biguanides, sulfonylureas, meglitinides, thiazolidinediones, and $\alpha$-glucosidase inhibitors. However, these treatments come with adverse effects such as flatulence, abdominal discomfort, or diarrhea (Phung et al., 2012). Natural substances with $\alpha$-glucosidase inhibitory activity in plants (Ashwini et al., 2015), vegetables (Sarkar et al., 2015), traditional medicines (Ren et al., 2015), and probiotics (Li et al., 2016a) are often effectively used to treat T2DM.

Probiotics from humans, animals, dairy products, and fermented foods are regarded as potential biotherapeutics for T2DM (Panwar et al., 2013), especially isolates from healthy people and those from natural dairy products. Oral administration of Lactobacillus paracasei TD062 in mice ameliorated the metabolism of lipids and glucose and reduced oxidative stress because of its $\alpha$-glucosidase inhibitory activity (Dang et al., 2018). Treatment with IL-10-inducing lactic acid bacteria alleviated metabolic disorders by suppressing chronic inflammation (Toshimitsu et al., 2016). Lacto- 
bacillus plantarum strain Ln4 showed inhibitory effects on obesity and insulin resistance by adipogenesis and glucose uptake in 3T3-L1 mice fed a high-fat diet (Lee et al., 2018). Several strains of lactic acid bacteria have been shown to attenuate the adverse effects of T2DM and obesity by preventing metabolic disorders and ameliorating insulin resistance by stimulating the immune system (Takemura et al., 2010; Xiao et al., 2014).

Enterococcus has shown a wide range of beneficial properties in vitro and in vivo (Hlivak et al., 2005; Cavallini et al., 2009; Guo et al., 2016). As an important species of nonstarter lactic acid bacteria in dairy products, it produces aromatic volatile compounds and bacteriocins against food spoilage or pathogenic bacteria (Listeria monocytogenes, Staphylococcus aureus, and Clostridium spp.) in a variety of cheeses during ripening (Giraffa, 2003). To date, most studies have focused on non-toxigenic E. faecium (Cavallini et al., 2009; Bhat and Bajaj, 2018; Hu et al., 2019) and E. faecalis (Mishra et al., 2019). Few studies have focused on E. hirae (Daillère et al., 2016), which has been isolated from fermented food (Sheoran and Tiwari, 2019), Brazilian artisanal cheese (Cavicchioli et al., 2019), and raw cow milk (Bouymajane et al., 2018), but seldom from humans. Daillère et al. (2016) observed that $E$. hirae and Barnesiella intestinihominis facilitated a cyclophosphamide-induced therapeutic immunomodulatory effect. Sheoran and Tiwari (2019) reported that bacteriocins from E. hirae LD3 and L. plantarum LD4 inhibited the growth of $S$. aureus in milk. However, to the best of our knowledge, the effect of E. hirae in chronic diseases, particularly T2DM, has not yet been reported.

In a previous study, we isolated E. hirae WEHI01 from a healthy Chinese infant (Zhang et al., 2017a). This strain is used in the fermentation of yogurt and has a wide range of beneficial properties, including cholesterol-lowering effects, high glycodeoxycholic acid-hydrolase activity, and strong adhesion capacity to Caco-2 cells (Zhang et al., 2017a). In this study, we further investigated the biological effects of $E$. $h i$ rae WEHI01, particularly its protective effect against T2DM in rat models of pre-insulin resistance induced by a high fat and sucrose (HFS) diet and T2DM induced by low-dose-streptozotocin and an HFS diet.

\section{MATERIALS AND METHODS}

\section{Animals and Experimental Design}

All procedures involving animals were approved by the Animal Care Review Committee (approval number 0064257) of Nanchang University, Jiangxi, China, and adhered to the institutional animal care committee guidelines.

Male Sprague-Dawley rats ( 5 wk old, 140-160 g) were acclimated for 1 wk using a standard diet and fresh water under a $12 \mathrm{~h}$ light/dark cycle at a temperature of $23 \pm 1^{\circ} \mathrm{C}$ and a humidity of $54 \pm 2 \%$. The experimental model is shown in Supplemental Figure S1 (https://doi .org/10.3168/jds.2019-17185). All rats were randomly assigned to 1 of 3 groups (9 rats per group): fed a normal chow diet (NDm); fed a HFS diet consisting of $10 \%$ lard, $10 \%$ sucrose, $55 \%$ corn starch, $20 \%$ casein, $1 \%$ vitamin mix, and $4 \%$ bone powder (Dm); WEHI01, fed the same diet as the Dm group plus E. hirae WEHI01 $\left(5.0 \times 10^{9} \mathrm{cfu} /\right.$ rat per day $)$. After $4 \mathrm{wk}, 3$ rats from each group (NDm, Dm, and WEHI01) were assigned as obesity models (normal chow, HFS, and HFS-WEHI01 groups, respectively). The remaining 6 rats from the NDm group were given PBS, and the remaining rats from the Dm and WEHI01 groups were given a tail vein injection of streptozotocin (dissolved in citrate buffer to $\mathrm{pH} 4.5,30 \mathrm{mg} / \mathrm{kg}$ of BW; Sigma, St. Louis, MO) to establish a T2DM model. We measured BW and 2-h postprandial blood glucose in all rats once per week.

\section{Measurement of Blood Glucose Levels}

To measure blood glucose levels, we conducted oral glucose tolerance tests (OGTT). Rats were fasted for $12 \mathrm{~h}$ and then administered glucose $2 \mathrm{~g} / \mathrm{kg}$ BW orally. Blood glucose levels were measured at 30,60, 90, and 120 min using a portable Contour blood glucose monitor (Ascensia Diabetes Care Shanghai Co. Ltd., Shanghai, China).

\section{Measurement of Blood Cytokines and Lipids}

On the final days of wk 4 and 9, animals were fasted for $12 \mathrm{~h}$ and anesthetized, and we collected blood samples from the orbital venous plexus and centrifuged them at $3,000 \times g$ at $4^{\circ} \mathrm{C}$ for $15 \mathrm{~min}$ to obtain the serum. We measured serum levels of tumor necrosis factor $\alpha$ (TNF- $\boldsymbol{\alpha}$ ), IL-6, and IL-10 using ELISA kits (Yuanye, Shanghai, China). Serum lipid concentrations, including total cholesterol (TC), triglycerides, high-density lipoprotein cholesterol (HDL-C), and low-density lipoprotein cholesterol (LDL-C), were evaluated at the Nanchang Qianmai Medical Laboratory Co., Ltd. (Jiangxi, China).

\section{Histopathological Evaluation}

At the end of the experiment, rat kidney, liver, and pancreas tissues were dissected, fixed with $4 \%$ para- 
formaldehyde solution, extracted, dehydrated, and embedded in paraffin. Then, 4-mm-thick samples were prepared, stained with hematoxylin and eosin, observed (Eclipse Ci, NIS_F_Ver46000_64bit_E; Nikon, Tokyo, Japan), and photographed under an optical microscope (Digital sight DS-FI2; Nikon).

\section{Total RNA Isolation and Quantitative RT-PCR Analysis}

Total RNA was extracted from the liver using the Takara MiniBEST Universal RNA Extraction Kit (Takara, Shiga, Japan), and cDNA was obtained by reverse transcription using the RevertAid First Strand cDNA Synthesis Kit (Thermo Scientific, Vilnius, Lithuania). The qPCR reactions were performed using a 7900HT fast real-time PCR system (Applied Biosystems, Foster City, CA) using SYBR Premix Ex Taq (Takara, Kusatsu, Japan). We used the housekeeping gene $A C T B$ for normalization. The sequences of primers (Invitrogen China, Ltd., Beijing, China) used for real-time quantitative PCR are shown in Table 1. Data analysis was carried out by using the $2^{-\Delta \Delta \mathrm{Ct}}$ method (Norris et al., 2017), and all gene transcript levels were standardized using the basic value of the HFS group.

\section{High-Throughput Sequencing and Bioinformatics Analysis}

The contents of the rats' ceca were collected and stored at $-80^{\circ} \mathrm{C}$ for use. Total DNA was extracted using the DNeasy Blood and Tissue Kit (Qiagen, Hilden, Germany) and used for 16S ribosomal (r)RNA gene amplicon sequencing on the Illumina MiSeq platform (BGI TechSolutions Co. Ltd., Shenzhen, China; Caporaso et al., 2012). The primers targeting the hypervariable V4 region of $16 \mathrm{~S}$ rRNA gene were designed as described by Pan et al., (2016).

The Quantitative Insights into Microbial Ecology pipeline was used for bioinformatics analysis of the sequencing data. We obtained clean reads from the raw data after removing contamination and low-quality data. Briefly, raw reads were blasted with the National Center for Biotechnology Information Sequence Read Archive database (https://www.ncbi.nlm.nih.gov/). High-quality clean reads were stitched into tags 252 bp long using Fast Length Adjustment of Short reads (FLASH, version 1.2.11; http://www.cbcb.umd.edu/ software/flash). Clean reads were filtered from raw reads to eliminate low-quality reads, and then highquality paired-end reads were combined with tags using FLASH. Sequences with $97 \%$ similarity were clustered to operational taxonomic units (OTU) at 97\% sequence similarity using UPARSE (http://www
Table 1. Gene primers used in the study

\begin{tabular}{|c|c|}
\hline Primer $^{1}$ & Oligonucleotide sequence $\left(5^{\prime}-3^{\prime}\right)$ \\
\hline Actin $\mathrm{F}$ & CACCATGTACCCAGGCATTG \\
\hline Actin R & ACAGTCCGCCTAGAAGCATT \\
\hline FMO3 F & CTCAGGTTGTGACATCGCTG \\
\hline FMO3 R & TGATCACCACCATGTCCCAA \\
\hline TNFA F & GGGCAGGTCTACTTTGGAGTCATTG \\
\hline TNFA R & GGGCTCTGAGGAGTAGACGATAAAG \\
\hline ChREBP F & CAGATGCGGGACATGTTTGA \\
\hline ChREBP R & AATAAAGGTCGGATGAGGATGCT \\
\hline CYP7A1 $\mathrm{F}$ & GATCCTGATGCTCTCCTGCT \\
\hline CYP7A1 R & CGCCAAGTGACATCATCCAG \\
\hline CRTC2 F & TGCCTACTGACCAGCGATTG \\
\hline CRTC2 $R$ & AATTGCTCCAGATTCCCCAGG \\
\hline$G K R P \mathrm{~F}$ & AAACGATATCAGCATGTGATCGA \\
\hline$G K R P \mathrm{R}$ & GCCGCTTCATACCCTGACA \\
\hline$L D L R \mathrm{~F}$ & TACTCTCTGGTGACCGAGGA \\
\hline$L D L R \mathrm{R}$ & GCTGGAGATGGAGTGGAGTT \\
\hline PPARA F & TCAAACTTGGGTTCCATGAT \\
\hline$P P A R A \mathrm{R}$ & TGAACAAAGACGGGATG \\
\hline
\end{tabular}

${ }^{1} \mathrm{~F}=$ forward; $\mathrm{R}=$ reverse.

.drive5.com/uparse/). We used Ribosomal Database Project Classifier version 2.2 (Wang et al., 2007) to analyze the phylogenetic affiliation of each $16 \mathrm{~S}$ rRNA gene sequence. Finally, we analyzed differences using $\alpha$-diversity and $\beta$-diversity. The $\alpha$-diversity analysis included indexes of Shannon diversity and Simpson diversity. The $\beta$-diversity analysis mainly explored the difference in microbial flora structure between samples, generally using principal component analysis (PCA). Principal components were developing using OTU abundance information, the relative abundance of each OTU in each sample was calculated, and then the PCA of OTU was conducted using the relative abundance values and ade4 (https://pbil.univ-lyon1.fr/CRAN/ web/packages/ade4/index.html) of software R (v. 3.1.1; https://cran.rstudio.com/).

\section{Statistical Analysis}

All experimental data were shown as mean \pm standard error of the mean (SEM). Multiple groups were tested using 1-way ANOVA and analyzed using 2-tailed unpaired Student's $t$ test. We considered $P<0.05$ as statistically significant. All statistical analyses were carried out using GraphPad Prism v5.01 (Graph Pad Software Inc., La Jolla, CA).

\section{RESULTS AND DISCUSSION}

\section{Effects of E. hirae WEHI01 on BW and Blood Glucose in Rats}

The intervention protocol investigating E. hirae WEHI01 in obesity and T2DM is summarized in Supplemental Figure S2 (https://doi.org/10.3168/jds.2019 
A

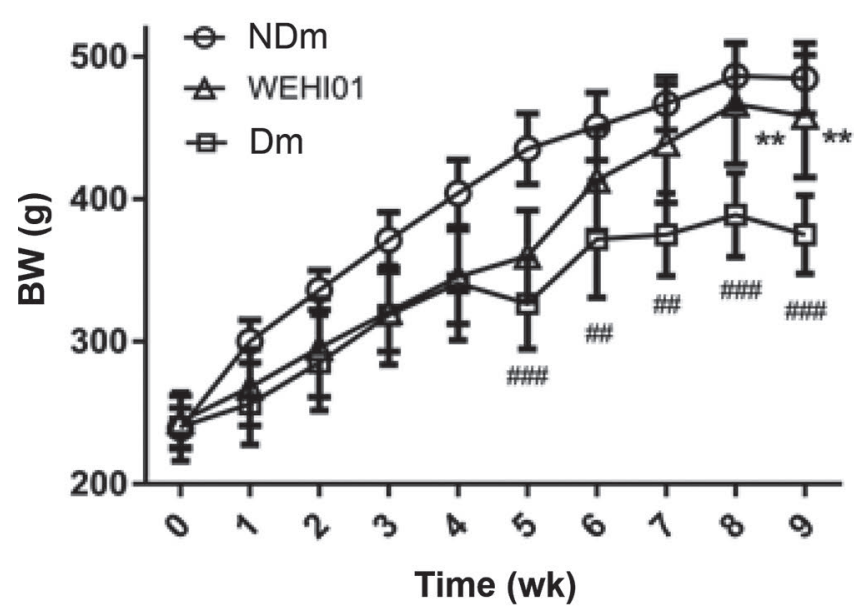

C

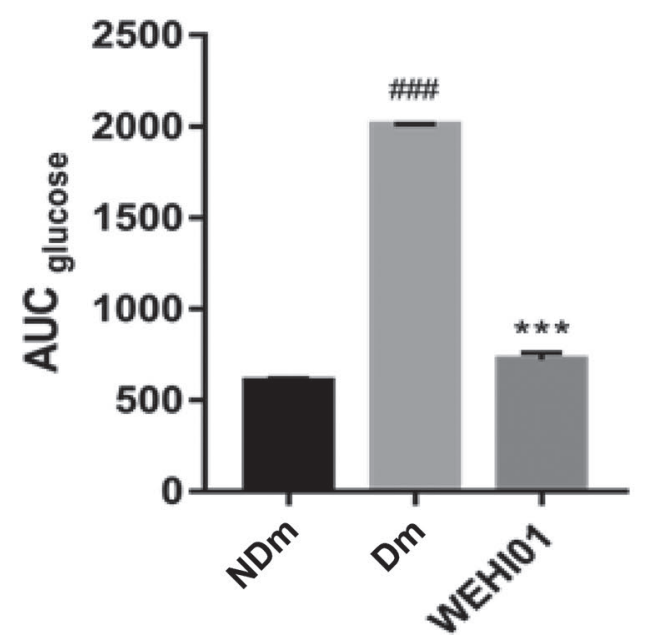

B

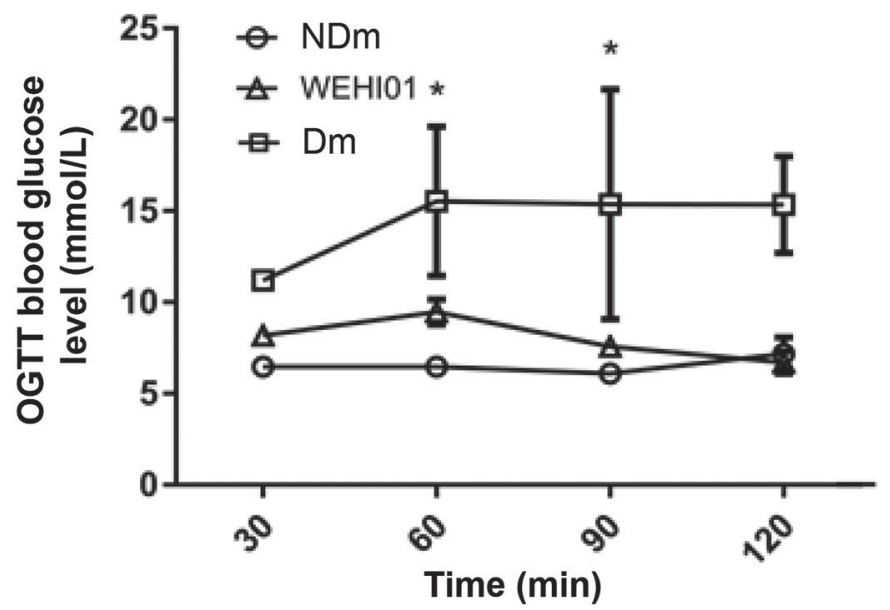

$\mathrm{D}$

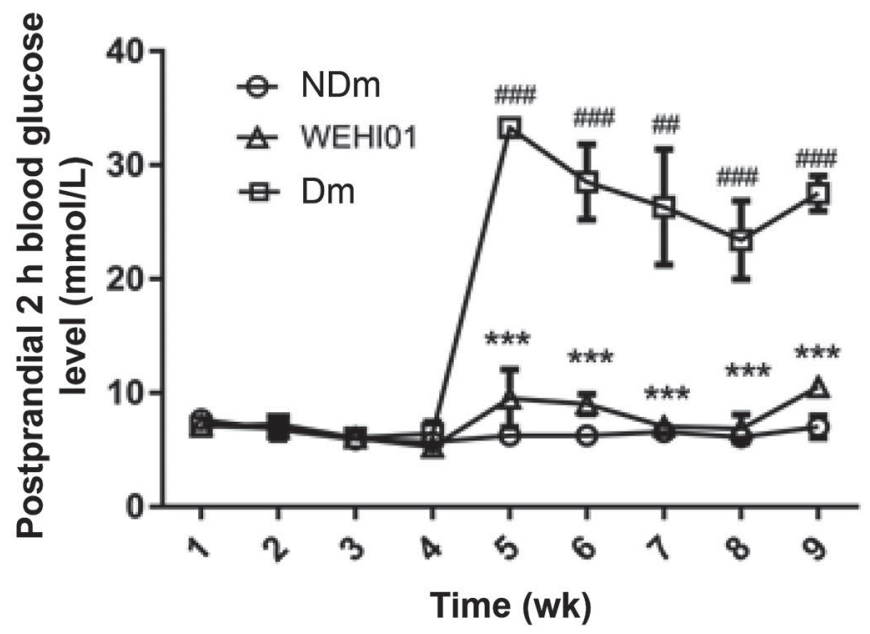

Figure 1. Effects of Enterococcus hirae WEHI01 on (A) BW; (B) oral glucose tolerance test (OGTT); (C) area under the curve of glucose $\left(\mathrm{AUC}_{\text {glucose }}\right)$; and (D) 2 -h postprandial blood glucose. Data are presented as mean \pm SEM. ${ }^{\# \# ~} P<0.01 ;{ }^{\# \#} P<0.001$ vs. NDm group; ${ }^{*} P<$ $0.05 ;{ }^{* *} P<0.01 ;{ }^{* * *} P<0.001$ vs. Dm group. Dm = rats with diabetes mellitus; $\mathrm{NDm}=$ rats without diabetes mellitus; WEHI01 $=$ rats with diabetes mellitus and supplemented with E. hirae WEHI01.

-17185), including indexes of BW and blood glucose levels. Streptozotocin has been widely used to induce a model of diabetes that is prone to weight loss due to abnormal carbohydrate and lipid metabolism, and increased catabolism of protein. As shown in Figure 1 , before injection of streptozotocin, the Dm and WEHI01 groups showed a similar tendency toward low $\mathrm{BW}$ compared to the NDm group; after injection of streptozotocin, the WEHI01 group demonstrated a significant increase in BW at 8 and 9 wk compared with the Dm group $(P<0.05)$. In contrast, the Dm group maintained a significantly low BW compared to the NDm group. Administration of E. hirae WEHI01 led to recovered BW, indicating that E. hirae WEHI01 might have a protective effect in terms of BW in T2DM. Still, it is important to note that in rats who received $E$. hirae WEHI01, BW was lower than in rats in the NDm group. This finding was consistent with a previous study (Chen et al., 2014a) showing that administration of L c casei CCFM0412 prevented excessive decreases in BW in mice with T2DM, but their BW was still lower than that of the control mice.

Glucose intolerance is a well-established diagnostic criterion for insulin resistance in T2DM. Glucose tolerance was impaired in the Dm group after streptozotocin injection. As shown in Figure 1B, administration 
of E. hirae WEHI01 led to OGTT levels at 60 and 90 min close to those of the NDm group, a significant decrease compared to the Dm group. Furthermore, taking the total area under the curve of glucose over $120 \mathrm{~min}$ as the quantitative result of the OGTT, the WEHI01 group showed a significant improvement in oral glucose tolerance (Figure 1C, $P<0.05$ ). Correspondingly, administration of $E$. hirae WEHI01 led lower postprandial blood glucose levels than the Dm group from $5 \mathrm{wk}$, levels similar to the NDm group. These results suggest that E. hirae WEHI01 can improve glucose tolerance in rats with $\mathrm{T} 2 \mathrm{DM}$ to some extent.

\section{Influence of E. hirae WEHI01 on Serum Lipid Levels in Rats}

As shown in Figure 2, TC and LDL-C levels in the HFS group were significantly higher than those in the normal chow group. However, TC, LDL-C, and HDL$\mathrm{C}$ levels in the HFS-WEHI01 group were significantly lower than those of the HFS group $(P<0.05)$. In addition to improving blood glucose status, E. hirae WEHI01 decreased serum TC, LDL-C, and HDL-C levels in obese rats, indicating the potential lipid-lowering activity of E. hirae WEHI01. Low-density lipoprotein cholesterol is the most dangerous factor in blood lipids because oxidized LDL-C is transported to the arterial wall to form atherosclerosis; the decreases in LDL-C we found suggest that E. hirae WEHI01 could play a role in alleviating atherosclerosis.

\section{Relative Expression of Genes Related to Inflammation, Blood Glucose, and Lipid Metabolism in Rat Liver}

Serum lipid and inflammatory levels may be partially affected by the expression of relevant genes in liver, such as CYPrA1, HMG-CoAS, SCD1, FAS, SCARB1, $L D L R$, and FMO3 (Huang et al., 2018). After lowering of serum lipid levels with E. hirae WEHI01, we also examined the expression of TNFA, CYP7A1, GKRP, ChREBP, CRTC2, PPARA, LDLR, and FMO3. As shown in Figure 3, transcription levels of $C Y P^{r} 7 A 1$ tended to be increased in the liver $(P>0.05)$, but levels of PPARA and $L D L R$ increased significantly ( $P$ $<0.01$ and $P<0.05$, respectively), and levels of TNFA, GKPP, CRTC2, and FMO3 decreased significantly $(P<0.05)$. Based on our findings and the results of previous studies, we propose the mechanism glycolipid metabolism regulation with E. hirae WEHI01 in the
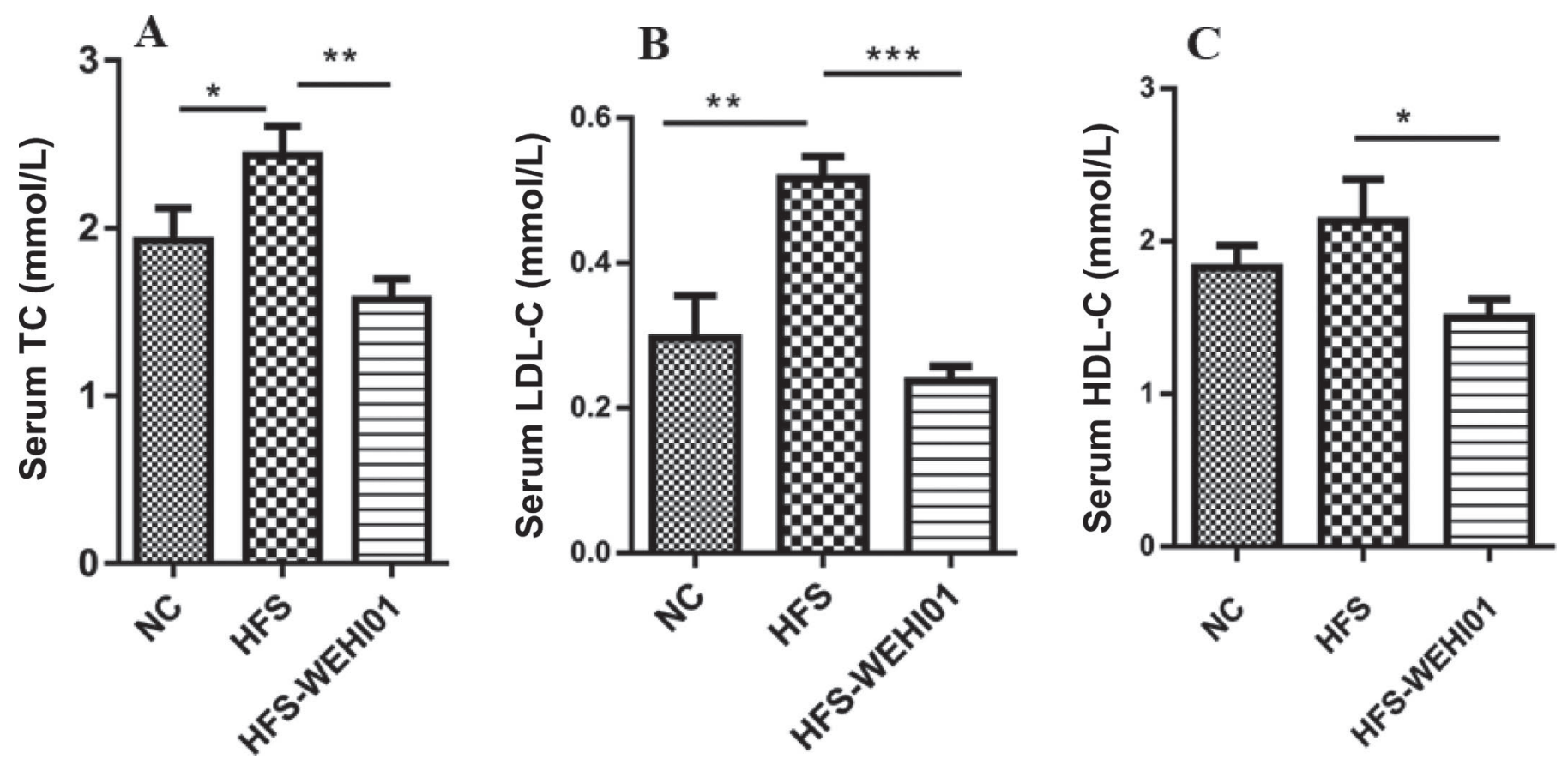

Figure 2. Effect of Enterococcus hirae WEHI01 on (A) serum total cholesterol (TC); (B) serum low-density lipoprotein cholesterol (LDL-C); and $(\mathrm{C})$ serum high-density lipoprotein cholesterol (HDL-C). HFS = high fat and sucrose diet; HFS-WEHI01 = high fat and sucrose diet combined with $E$. hirae WEHI01; NC $=$ normal chow diet. Values are expressed as mean $\pm \mathrm{SEM}\left(\mathrm{n}=3\right.$ per group). ${ }^{*} P<0.05 ; * * P<0.01 ;{ }^{* * *} P$ $<0.001$. 


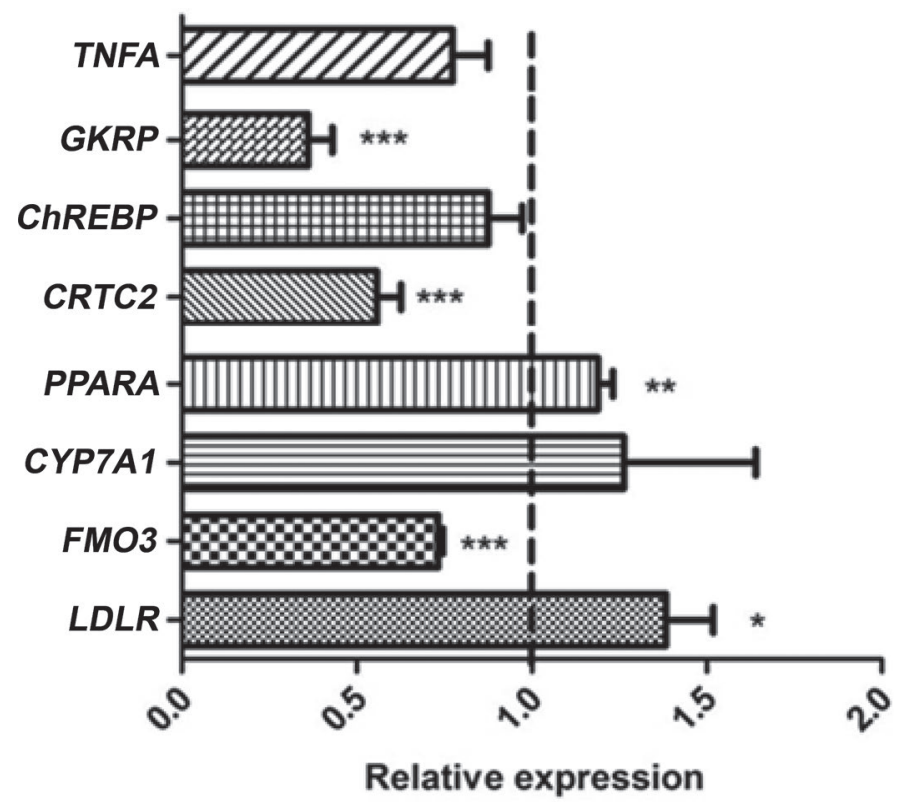

Figure 3. Relative expression of genes related to inflammation, blood glucose, and lipid metabolism in the liver of rats by quantitative PCR analysis using GAPDH as the internal reference. Relative expression indicates the group fed a high fat and sucrose diet combined with Enterococcus hirae WEHI01 (HFS-WEHI01) compared with the group fed a high fat and sucrose diet only (HFS). Values are expressed as mean \pm SEM $\left(\mathrm{n}=3\right.$ per group). ${ }^{*} P<0.05 ;{ }^{* *} P<0.01 ; * * * P<0.001$.

liver of obese mice as follows: E. hirae WEHI01 significantly upregulated transcription levels of PPARA $(P<0.01)$, accelerating the metabolism of total bile acids and significantly downregulating ChREBP and CRTC2 $(P<0.001)$ to promote sugar metabolism and reduce fat accumulation. For cholesterol metabolism, downregulation of FMO3 $(P<0.001)$ coupled with an increase in $C Y P 7 A 1$ might contribute to a reduction in yield of TMAO, alleviating the negative feedback regulation of cholesterol 7-hydroxylase to accelerate cholesterol decomposition, as suggested by our previous study (Huang et al., 2018).

\section{Influence of E. hirae WEHI01 on Serum Inflammatory Cytokines in Rats with T2DM}

Type 2 diabetes mellitus is a common complication in obesity, characterized by low-grade inflammation (Burcelin et al., 2011) and higher levels of proinflammatory cytokines in serum (Tian et al., 2016). Consistent with a previous report (Wang et al., 2017), we observed that E. hirae WEHI01 significantly reduced serum levels of IL-6 $(P<0.05)$, IL-10 $(P<0.05)$, and TNF- $\alpha(P<$ $0.01)$ in the WEHI01 group compared with the Dm group (Figure 4). Interleukin-6 acts on islets during onset of T2DM, and high concentrations of IL-6 inhibit insulin secretion (Rabinovitch, 1998). Tumor necrosis factor $\alpha$ interacts with other cytokines to affect insulin secretion (Liu et al., 2016), and plays an important role in the development of T2DM (Mishima et al., 2001). In contrast, IL-10 plays a vital role in the regulation of immune response and inflammatory response (Toshimitsu et al., 2016), and elevated levels in people with diabetes may be a precursor to hypertension (Zietz et al., 2000). Consistent with evidence that L. casei CCFM419 reduces inflammatory cytokines levels in mice with diabetes ( $\mathrm{Li}$ et al., 2016b), administration
A

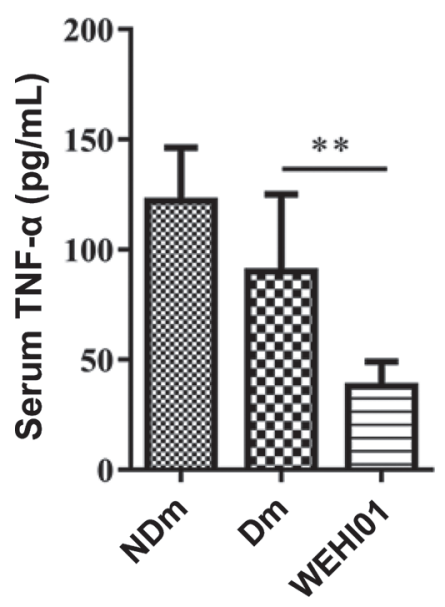

B

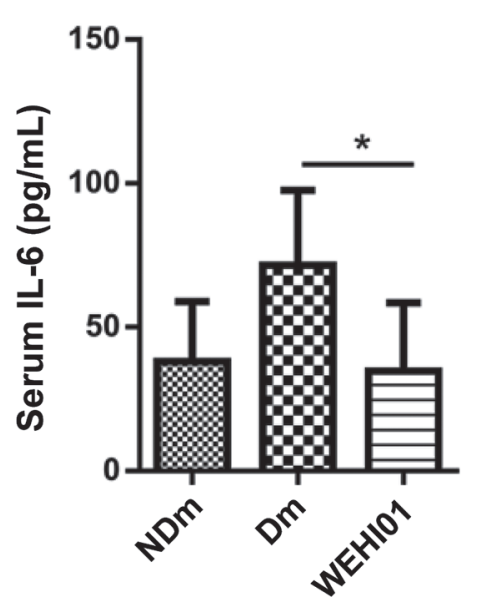

C

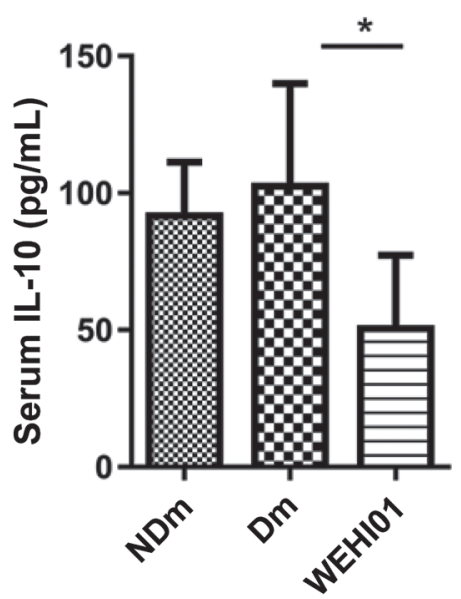

Figure 4. Effect of Enterococcus hirae WEHI01 on serum (A) tumor necrosis factor $\alpha$ (TNF- $\alpha$ ); (B) IL-6; and (C) IL-10 in each group. Values are expressed as mean \pm SEM $\left(\mathrm{n}=5\right.$ per group). ${ }^{*} P<0.05 ; * *<0.01$. Dm $=$ rats with diabetes mellitus; NDm $=$ rats without diabetes mellitus; WEHI01 = rats with diabetes mellitus and supplemented with E. hirae WEHI01. 
of E. hirae WEHI01 exhibited the potential to alleviate inflammation in rats with T2DM.

\section{Effect of E. hirae WEHI01 on Histological Changes in Rats with T2DM}

To confirm improvements in the syndromes of T2DM with E. hirae WEHI01, we performed histological analysis of the pancreas (Figure 5A), kidney (Figure 5B), and liver (Figure 5C) of the rats in the study. The NDm group showed normal proportions and normal structure of the islets of Langerhans. Acinar cells stained strongly and were arranged closely. We observed a remarkable difference between the Dm and NDm groups with respect to the shape of the pancreas and the number of islets of Langerhans, suggesting necrosis of some acinar cells around the pancreatic islet. Importantly, oral administration of E. hirae WEHI01 led to partial restoration of islet cells and decreased $\beta$-cell necrosis and vacuolation (Figure 5A). This finding was consistent with the results of a previous study ( $\mathrm{Li}$ et al., 2016b), which demonstrated that administration of $L$. casei CCFM419 and dead L. casei CCFM419 protected against pancreatic impairment, and the extent of cellular repair was greater with L. casei CCFM419 than with dead L. casei CCFM419.

Similarly, destruction of renal tissue (e.g., more renal tubular epithelial cell necrosis, deep staining or fragmentation of the nucleus pyknosis and glomerulus mild congestion) in the Dm group was reversed by E. hirae WEHI01 treatments in the WEHI01 group (Figure 5B); namely, some renal tubular epithelial cells were shed into the lumen, and this renal injury was alleviated.

A
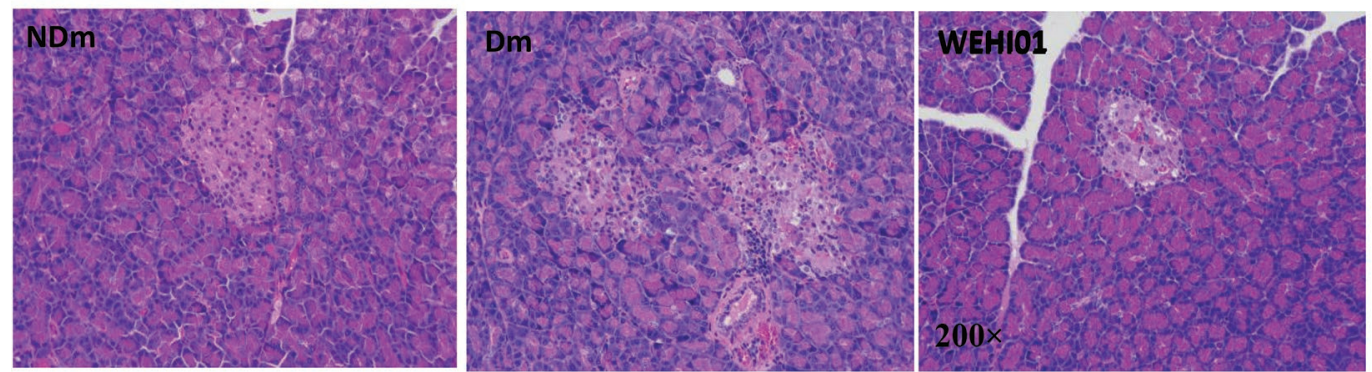

B
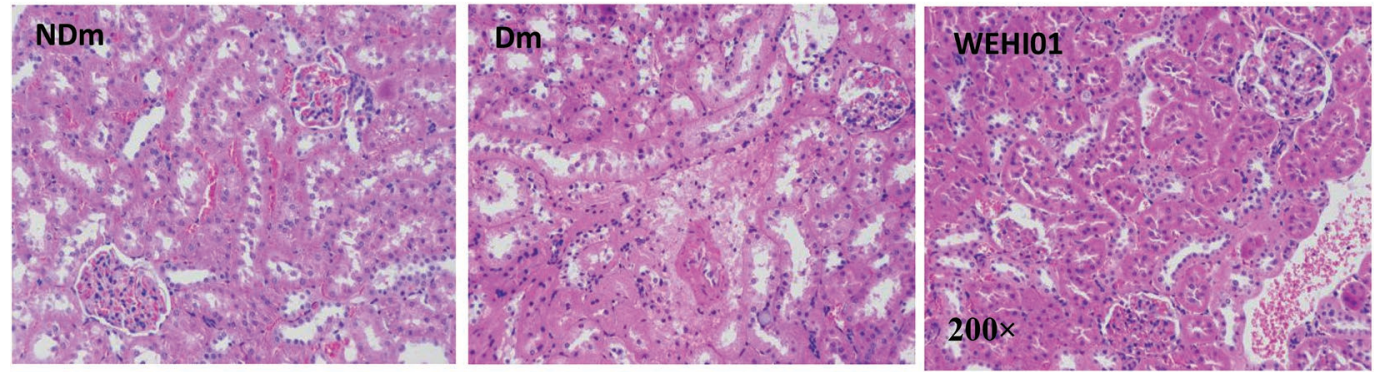

C
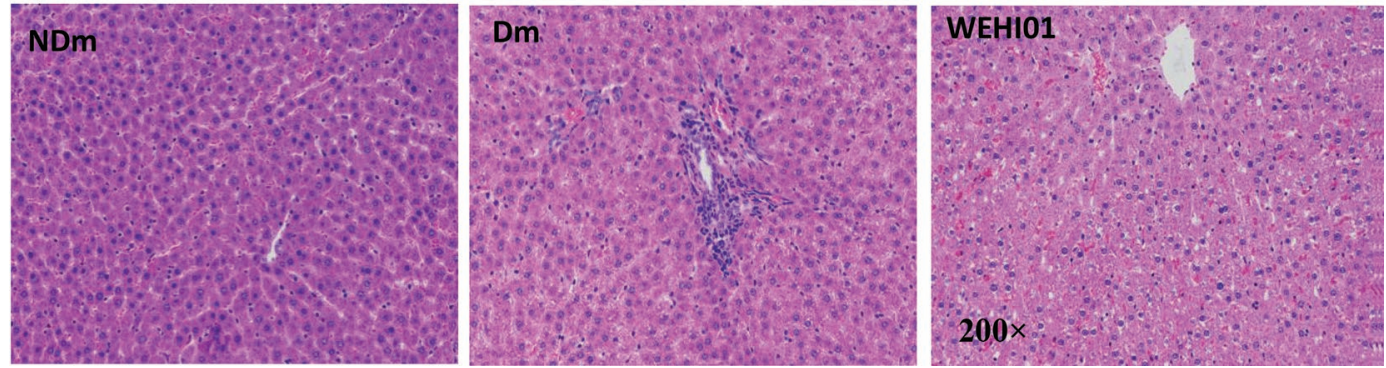

Figure 5. Effect of Enterococcus hirae WEHI01 on histology (200x) of the (A) pancreas, (B) kidney, and (C) liver in rats in the NDm, Dm, and WEHI01 groups. Hematoxylin and eosin staining. Dm = rats with diabetes mellitus; NDm $=$ rats without diabetes mellitus; WEHI01 = rats with diabetes mellitus and supplemented with E. hirae WEHI01. 
In the liver (Figure 5C), hepatocyte edema and hepatic sinus congestion were greatly reduced in liver tissue after the administration of E. hirae WEHI01.

\section{Effect of E. hirae WEHI01 on Total Bile Acid Levels in Rats}

An increase in the expression of PPARA and $C Y P$ Y $A 1$ in the obese model (Figure 3) might have promoted the conversion of cholesterol into bile acid, reducing cholesterol content; as shown in Figure 6, E. hirae WEHI01 significantly reduced serum total bile acid levels $(P<$ 0.001) compared to the Dm group. This finding was supported by a similar study (Jiang et al., 2018). High bile acid levels are risk factors for T2DM (Sheoran and Tiwari, 2019), and their regulation in vivo may be a potential target for the treatment of metabolic diseases (Thomas et al., 2008).

\section{Effect of E. hirae WEHI01 on Intestinal Microbiota of Rats}

Prawitt et al. (2011) proposed that the pathogenesis of T2DM might be due to bile-acid-mediated control, which in turn is related to changes in microflora. To assess whether E. hirae WEHI01 changed the gut microbial community in rats with T2DM, we sequenced the 16S rRNA genes from variable regions V4 of feces in rats using Illumina HiSeq/MiSeq platforms. As shown in Figure 7A, the intestinal microbiota among the 3 study groups were obviously different. The distance of 2 data points in the 2-dimensional PCA plot is a visual representation of the similarity between different microbiota compositions (Tian et al., 2016). The Dm group had a distinct microbiota composition that was clustered differently from the other 2 groups. As shown in Figure 7B, the community richness of the Dm group measured by $\alpha$ diversity was increased (Shannon index) and decreased (Simpson index) at the OTU level compared with the NDm group, and the WEHI01 group showed a lower tendency toward an increase in diversity of intestinal microbiota.

To profile specific changes in the gut microbiota, we also analyzed the relative abundance of predominant taxa in the 3 groups. Figure 8 shows a detailed overview of the cecal microbiota composition at the level of phylum, class, order, and family. Compared with the Dm group, we found a nonsignificant difference in dominant phyla, including Firmicutes and Bacteroidetes, for the WEHI01 group $(P>0.05)$, but the abundance of Actinobacteria and Verrucomicrobia were significantly decreased $(P<0.01$ and $P<0.05$, respectively). At the class level, Bacilli and Verrucomicrobiae were significantly increased and decreased in the WEHI01 group, respectively $(P<0.05)$. At the order level, the abundance of Lactobacillales was significantly increased in the WEHI01 group $(P<0.05)$. At the family level, the abundance of Lactobacillaceae was significantly increased, and that of Ruminococcaceae was significantly decreased for WEHI01 group $(P<0.05)$.

Gut microbiota play an important role in the development of T2DM (Cani et al., 2012). To the best of our knowledge, the phyla that account for the vast majority of all gut microbes include the gram-negative Bacteroidetes, Proteobacteria, and Verrucomicrobia, and the gram-positive Firmicutes and Actinobacteria (Kootte et al., 2012). Type 2 diabetes mellitus is characterized by a moderate degree of gut microbial dysbiosis, a decrease in the abundance of some universal butyrate-producing bacteria and an increase in various opportunistic pathogens (Qin et al., 2012). For ex-

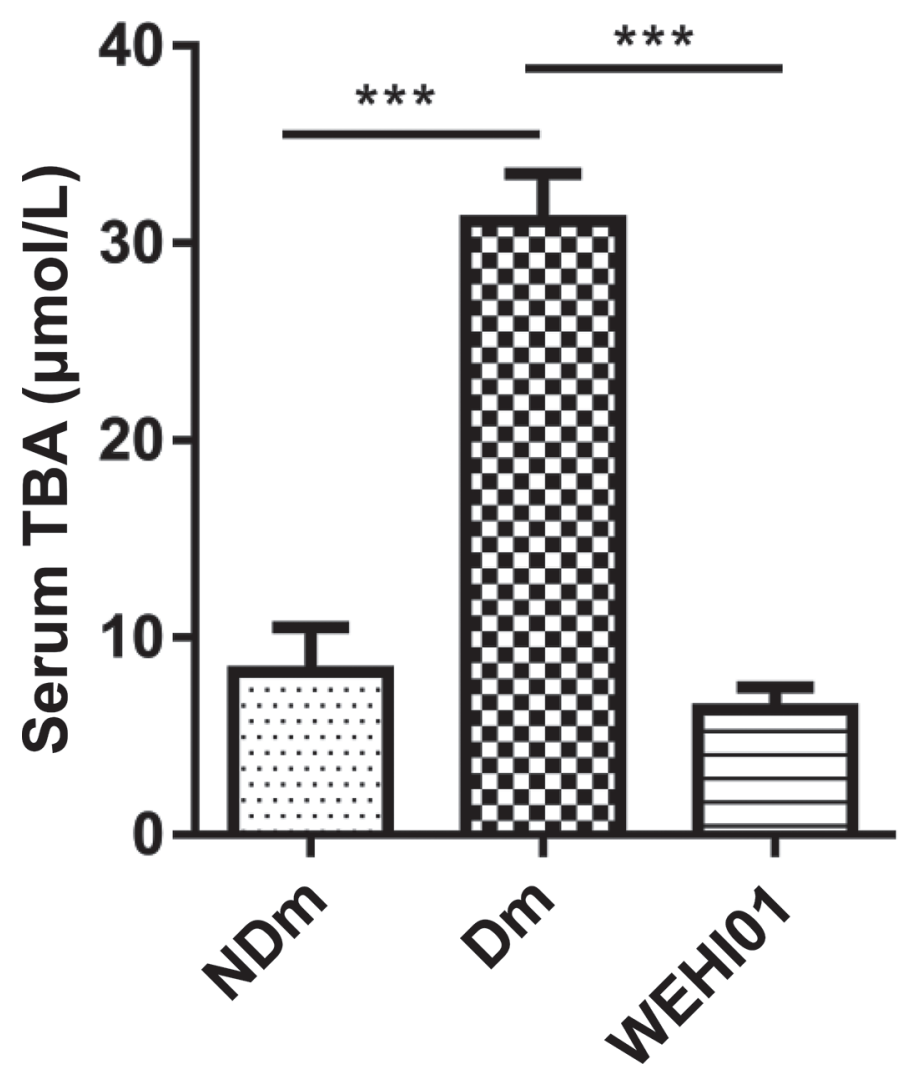

Figure 6. Effect of Enterococcus hirae WEHI01 on total bile acid (TBA) levels in the serum of each group. Values are expressed as mean \pm SEM ( $\mathrm{n}=5$ per group). ${ }^{* * *} P<0.001 . \mathrm{Dm}=$ rats with diabetes mellitus; NDm = rats without diabetes mellitus; WEHI01 = rats with diabetes mellitus and supplemented with E. hirae WEHI01. 
ample, several studies have suggested that obesity and T2DM are associated with an increase in Firmicutes and a lower abundance of Bacteroidetes (Turnbaugh et al., 2009; Zhang et al., 2013). The antidiabetic ef- fect of E. hirae WEHI01 may be partially mediated by regulating the composition of gut microbes. In the current study, E. hirae WEHI01 could have improved T2DM by changing the proportion of Bacteroidetes to
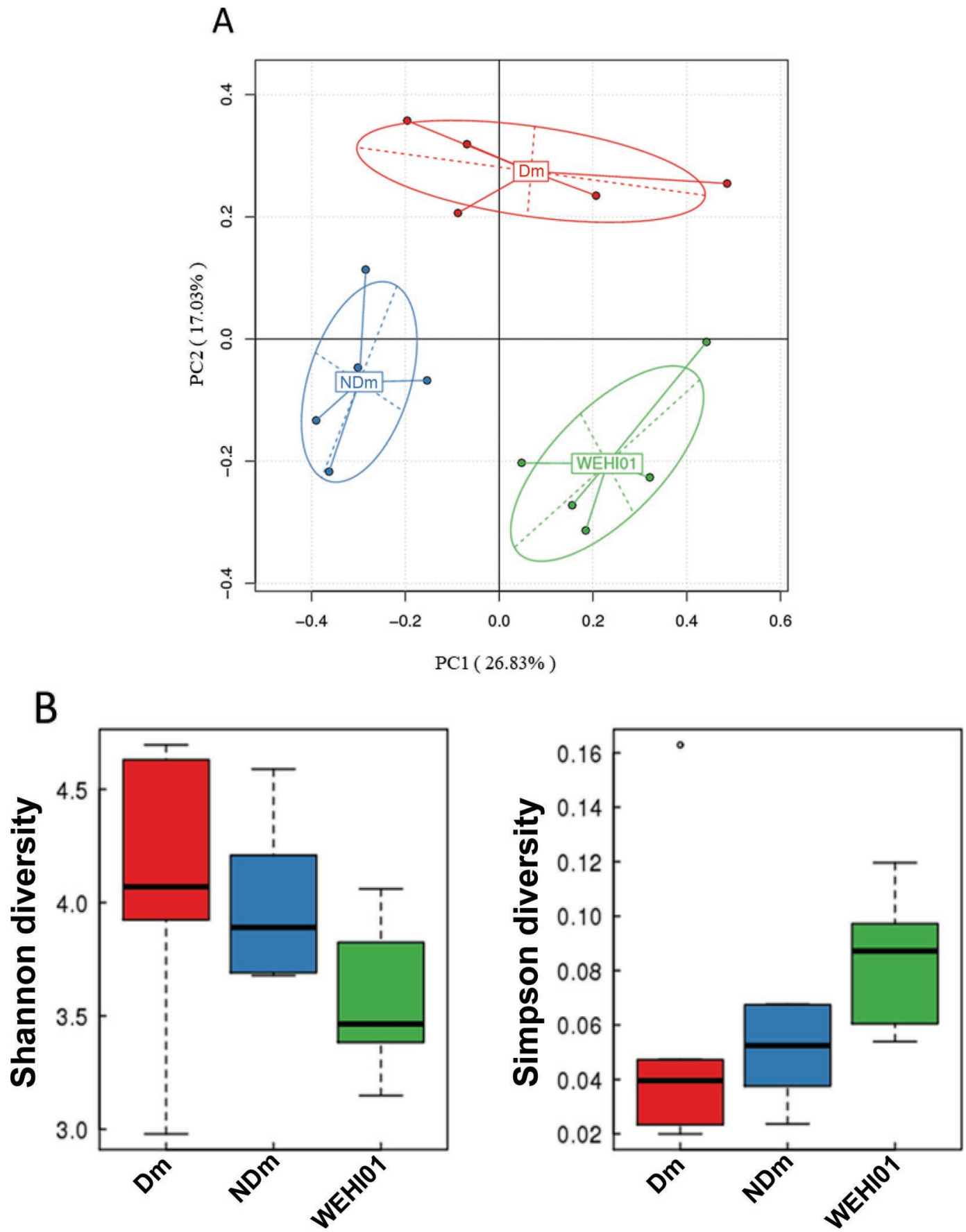

Figure 7. Effect of Enterococcus hirae WEHI01 on intestinal microbiota. (A) Principal component analysis (PCA), and (B) Shannon diversity and Simpson diversity. Boxplots were used to display the differences in $\alpha$-diversity among groups. The 5 horizontal lines, from bottom to top, are the minimum value, the first quartile, median, the third quartile, and the maximum value, respectively. Circles indicate outliers. Dm $=$ rats with diabetes mellitus; NDm = rats without diabetes mellitus; WEHI01 = rats with diabetes mellitus and supplemented with E. hirae WEHI01. 
Phylum

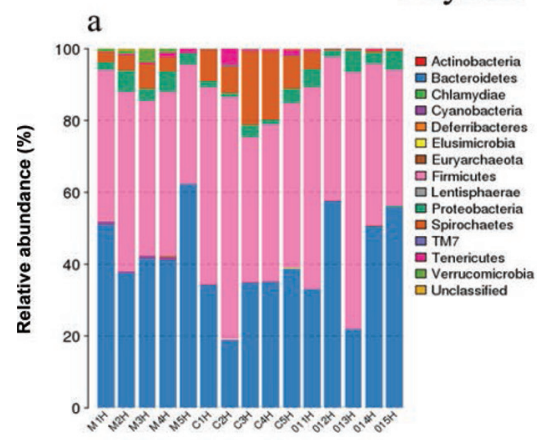

$\mathrm{b}$

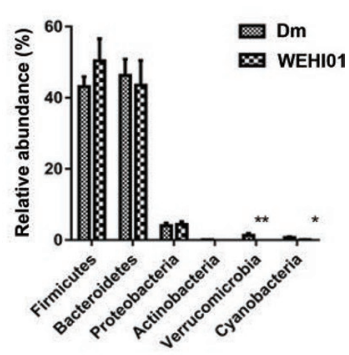

Class
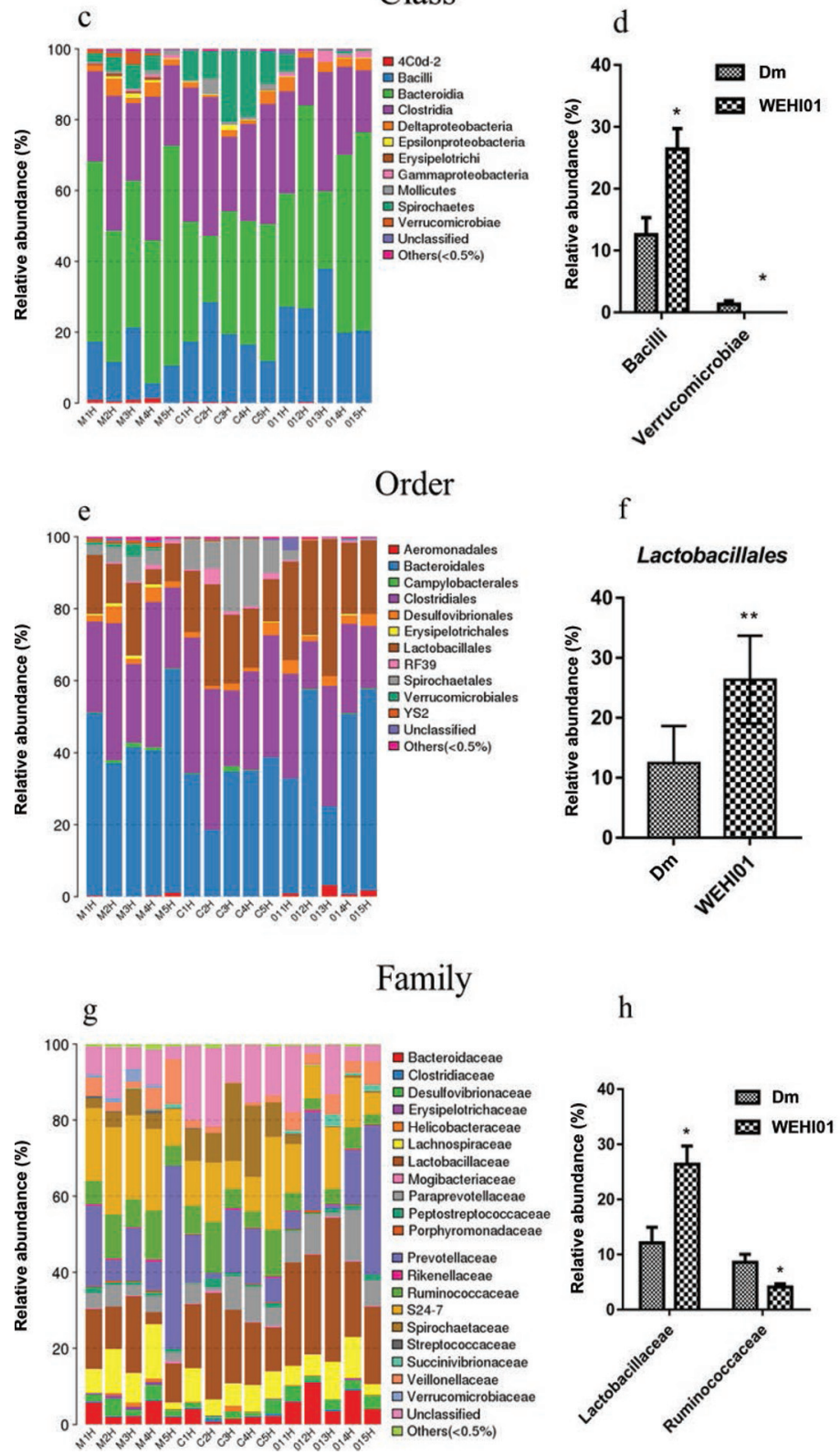

Figure 8. Effect of Enterococcus hirae WEHI01 on relative abundance at phylum, class, order, and family levels of the intestinal microbiota. ${ }^{*} P<0.05 ;{ }^{* *} P<0.01$ compared with Dm group. Dm $=$ rats with diabetes mellitus $(\mathrm{M} 1 \mathrm{H}-\mathrm{M} 5 \mathrm{H}$ on $\mathrm{x}$-axis of figures); NDm $=$ rats without diabetes mellitus $(\mathrm{C} 1 \mathrm{H}-\mathrm{C} 5 \mathrm{H})$; WEHI01 = rats with diabetes mellitus and supplemented with E. hirae WEHI01 (011H-015H). Error bars indicate SD. 
Firmicutes and increasing the abundance of Lactobacillales. Similarly, L. casei CCFM419 improved T2DM by changing the ratio of Bacteroidetes to Firmicutes and enriching the abundance of Lactobacillales (Zhang et al., 2013). Moreover, information in the literature varies with respect to the benefits of Lactobacillaceae (such as improving T2DM, anti-inflammatory effects, and reduced BW; Yadav et al., 2007; Kang et al., 2010), by producing short-chain fatty acids, which could be helpful for energy metabolism, maintaining intestinal integrity, preventing endotoxemia, and intestinal gluconeogenesis (Zhang et al., 2017b).

Some publications have reported on the administration of non- E. hirae strains. For instance, L. casei reduced obesity in rats with obesity and T2DM (Zhang et al., 2014). To the best of our knowledge, we have reported for the first time that E. hirae from an infant improved obesity and $\mathrm{T} 2 \mathrm{DM}$ in rats.

\section{CONCLUSIONS}

Our study demonstrated that the administration of E. hirae WEHI01 could lead to recovery of BW loss and improvements in serum lipids, regulate glycolipid metabolism in the liver associated with HFS-induced obesity, and improve glucose tolerance in a T2DM rat model. It also reduced levels of inflammation and bile acids in serum, and restored the morphology of the pancreas, kidney, and liver. Moreover, E. hirae WEHI01 dramatically modified the gut microbiota and increased the abundance of Lactobacillales. Our findings suggest that E. hirae WEHI01 isolated from a Chinese healthy infant has potential as a therapeutic probiotic for treating obesity and even T2DM by altering the gut microbiome.

\section{ACKNOWLEDGMENTS}

This study was sponsored by the National Nature Science Foundation of China (grant no. NSF 31570075, 31800037 and 31770133; Beijing, China), Jiangxi Province Science Foundation for Youths (Jiangxi, China; 20171BAB214001) and Natural Science Foundation of Jiangxi (no. 2016BAB215206). The authors have not stated any conflicts of interest.

\section{REFERENCES}

Ashwini, S., Z. Bobby, M. Joseph, S. E. Jacob, and R. Padmapriya. 2015. Insulin plant (Costus pictus) extract improves insulin sensitivity and ameliorates atherogenic dyslipidaemia in fructose induced insulin resistant rats: Molecular mechanism. J. Funct. Foods 17:749-760. https://doi.org/10.1016/j.jff.2015.06.024.

Bhat, B., and B. K. Bajaj. 2018. Hypocholesterolemic and bioactive potential of exopolysaccharide from a probiotic Enterococcus fae- cium K1 isolated from kalarei. Bioresour. Technol. 254:264-267. https://doi.org/10.1016/j.biortech.2018.01.078.

Bouymajane, A., F. Rhazi Filali, S. Oulghazi, A. Ed-dra, F. Benhallam, A. El Allaoui, J. Anissi, K. Sendide, B. Ouhmidou, and M. Moumni. 2018. Occurrence, molecular and antimicrobial resistance of Enterococcus spp. isolated from raw cow's milk trade by street trading in Meknes city, Morocco. Germs 8:77-84. https://doi.org/ 10.18683/germs.2018.1134.

Burcelin, R., M. Serino, C. Chabo, V. Blasco-Baque, and J. Amar. 2011. Gut microbiota and diabetes: From pathogenesis to therapeutic perspective. Acta Diabetol. 48:257-273. https://doi.org/10 .1007/s00592-011-0333-6.

Cani, P. D., M. Osto, L. Geurts, and A. Everard. 2012. Involvement of gut microbiota in the development of low-grade inflammation and type 2 diabetes associated with obesity. Gut Microbes 3:279-288. https://doi.org/10.4161/gmic.19625.

Caporaso, J. G., C. L. Lauber, W. A. Walters, D. Berg-Lyons, J. Huntley, N. Fierer, S. M. Owens, J. Betley, L. Fraser, M. Bauer, N. Gormley, J. A. Gilbert, G. Smith, and R. Knight. 2012. Ultrahigh-throughput microbial community analysis on the Illumina HiSeq and MiSeq platforms. ISME J. 6:1621-1624. https://doi .org/10.1038/ismej.2012.8.

Cavallini, D. C., R. Bedani, L. Q. Bomdespacho, R. C. Vendramini, and E. A. Rossi. 2009. Effects of probiotic bacteria, isoflavones and simvastatin on lipid profile and atherosclerosis in cholesterol-fed rabbits: A randomized double-blind study. Lipids Health Dis. 8:1. https://doi.org/10.1186/1476-511X-8-1.

Cavicchioli, V. Q., S. D. Todorov, I. Iliev, I. Ivanova, D. Drider, and L. A. Nero. 2019. Physiological and molecular insights of bacteriocin production by Enterococcus hirae ST57ACC from Brazilian artisanal cheese. Braz. J. Microbiol. 50:369-377. https://doi.org/ 10.1007/s42770-019-00068-4.

Chen, P., Q. Zhang, H. Dang, X. Liu, F. Tian, J. Zhao, Y. Chen, H. Zhang, and W. Chen. 2014a. Antidiabetic effect of Lactobacillus casei CCFM0412 on mice with type 2 diabetes induced by a highfat diet and streptozotocin. Nutrition 30:1061-1068. https://doi .org/10.1016/j.nut.2014.03.022.

Chen, P., Q. Zhang, H. Dang, X. Liu, F. Tian, J. Zhao, Y. Chen, H. Zhang, and W. Chen. 2014b. Oral administration of Lactobacillus rhamnosus CCFM0528 improves glucose tolerance and cytokine secretion in high-fat-fed, streptozotocin-induced type 2 diabetic mice. J. Funct. Foods 10:318-326. https://doi.org/10.1016/j.jff .2014.06.014.

Cho, N. H., J. E. Shaw, S. Karuranga, Y. Huang, J. D. da Rocha Fernandes, A. W. Ohlrogge, and B. Malanda. 2018. IDF Diabetes Atlas: Global estimates of diabetes prevalence for 2017 and projections for 2045. Diabetes Res. Clin. Pract. 138:271-281. https://doi .org/10.1016/j.diabres.2018.02.023.

Daillère, R., M. Vétizou, N. Waldschmitt, T. Yamazaki, C. Isnard, V. Poirier-Colame, C. P. Duong, C. Flament, P. Lepage, M. P. Roberti, B. Routy, N. Jacquelot, L. Apetoh, S. Becharef, S. Rusakiewicz, P. Langella, H. Sokol, G. Kroemer, D. Enot, A. Roux, A. Eggermont, E. Tartour, L. Johannes, P.-L. Woerther, E. Chachaty, J.-C. Soria, E. Golden, S. Formenti, M. Plebanski, M. Madondo, P. Rosenstiel, D. Raoult, V. Cattoir, I. G. Boneca, M. Chamaillard, and L. Zitvogel. 2016. Enterococcus hirae and Barnesiella intestinihominis facilitate cyclophosphamide-induced therapeutic immunomodulatory effects. Immunity 45:931-943. https://doi.org/ 10.1016/j.immuni.2016.09.009.

Dang, F., Y. Jiang, R. Pan, Y. Zhou, S. Wu, R. Wang, K. Zhuang, W. Zhang, T. Li, and C. Man. 2018. Administration of Lactobacillus paracasei ameliorates type 2 diabetes in mice. Food Funct. 9:3630-3639. https://doi.org/10.1039/C8FO00081F.

Fujimoto, W. Y. 2000. The importance of insulin resistance in the pathogenesis of type 2 diabetes mellitus. Am. J. Med. 108:9S-14S. https://doi.org/10.1016/S0002-9343(00)00337-5.

Giraffa, G. 2003. Functionality of enterococci in dairy products. Int. J. Food Microbiol. 88:215-222. https://doi.org/10.1016/S0168 $-1605(03) 00183-1$

Guo, L., T. Li, Y. Tang, L. Yang, and G. Huo. 2016. Probiotic properties of Enterococcus strains isolated from traditional naturally 
fermented cream in China. Microb. Biotechnol. 9:737-745. https:/ /doi.org/10.1111/1751-7915.12306.

Hlivak, P., J. Odraska, M. Ferencik, L. Ebringer, E. Jahnova, and Z. Mikes. 2005. One-year application of probiotic strain Enterococcus faecium M-74 decreases serum cholesterol levels. Bratisl. Lek Listy 106:67-72.

Hu, C., W. Xing, X. Liu, X. Zhang, K. Li, J. Liu, B. Deng, J. Deng, Y. Li, and C. Tan. 2019. Effects of dietary supplementation of probiotic Enterococcus faecium on growth performance and gut microbiota in weaned piglets. AMB Express 9:33. https://doi.org/ 10.1186/s13568-019-0755-Z.

Huang, F., F. Zhang, D. Xu, Z. Zhang, F. Xu, X. Tao, L. Qiu, and H. Wei. 2018. Enterococcus faecium WEFA23 from infants lessens high-fat-diet-induced hyperlipidemia via cholesterol 7-alphahydroxylase gene by altering the composition of gut microbiota in rats. J. Dairy Sci. 101:7757-7767. https://doi.org/10.3168/jds .2017-13713.

Intagliata, N., and K. L. Koch. 2007. Gastroparesis in type 2 diabetes mellitus: Prevalence, etiology, diagnosis, and treatment. Curr. Gastroenterol. Rep. 9:270-279. https://doi.org/10.1007/s11894 -007-0030-3.

Jiang, Y., C. Fu, G. Liu, J. Guo, and Z. Su. 2018. Cholesterol-lowering effects and potential mechanisms of chitooligosaccharide capsules in hyperlipidemic rats. Food Nutr. Res. 62. https://doi.org/10 $.29219 /$ fnr.v62.1446.

Kang, J.-H., S.-I. Yun, and H.-O. Park. 2010. Effects of Lactobacillus gasseri BNR17 on body weight and adipose tissue mass in diet-induced overweight rats. J. Microbiol. 48:712-714. https://doi .org/10.1007/s12275-010-0363-8.

Kootte, R. S., A. Vrieze, F. Holleman, G. M. Dallinga-Thie, E. G. Zoetendal, W. M. de Vos, A. K. Groen, J. B. L. Hoekstra, E. S. Stroes, and M. Nieuwdorp. 2012. The therapeutic potential of manipulating gut microbiota in obesity and type 2 diabetes mellitus. Diabetes Obes. Metab. 14:112-120. https://doi.org/10.1111/j.1463 $-1326.2011 .01483 . x$

Lee, E., S.-R. Jung, S.-Y. Lee, N.-K. Lee, H.-D. Paik, and S.-I. Lim. 2018. Lactobacillus plantarum strain Ln4 attenuates diet-induced obesity, insulin resistance, and changes in hepatic mRNA levels associated with glucose and lipid metabolism. Nutrients 10:643. https://doi.org/10.3390/nu10050643.

Li, X., N. Wang, B. Yin, D. Fang, J. Zhao, H. Zhang, and W. Chen. 2016a. Lactobacillus plantarum X1 with $\alpha$-glucosidase inhibitory activity ameliorates type 2 diabetes in mice. RSC Advances 6:63536-63547.

Li, X., Q. Xu, T. Jiang, S. Fang, G. Wang, J. Zhao, H. Zhang, and W. Chen. 2016b. A comparative study of the antidiabetic effects exerted by live and dead multi-strain probiotics in the type 2 diabetes model of mice. Food Funct. 7:4851-4860. https://doi.org/10 $.1039 / \mathrm{C} 6 \mathrm{FO} 01147 \mathrm{~K}$.

Liu, C., X. Feng, Q. Li, Y. Wang, Q. Li, and M. Hua. 2016. Adiponectin, TNF- $\alpha$ and inflammatory cytokines and risk of type 2 diabetes: A systematic review and meta-analysis. Cytokine 86:100-109. https://doi.org/10.1016/j.cyto.2016.06.028.

Mishima, Y., A. Kuyama, A. Tada, K. Takahashi, T. Ishioka, and M. Kibata. 2001. Relationship between serum tumor necrosis factor-a and insulin resistance in obese men with Type 2 diabetes mellitus. Diabetes Res. Clin. Pract. 52:119-123. https://doi.org/10.1016/ S0168-8227(00)00247-3.

Mishra, A. K., S. S. Kumar, and A. R. Ghosh. 2019. Probiotic Enterococcus faecalis AG5 effectively assimilates cholesterol and produces fatty acids including propionate. FEMS Microbiol. Lett. 366. https://doi.org/10.1093/femsle/fnz039.

Norris, G. H., C. M. Porter, C. Jiang, C. L. Millar, and C. N. Blesso. 2017. Dietary sphingomyelin attenuates hepatic steatosis and adipose tissue inflammation in high-fat-diet-induced obese mice. J. Nutr. Biochem. 40:36-43.

Pan, M., C. Wan, Q. Xie, R. Huang, X. Tao, N. P. Shah, and H. Wei. 2016. Changes in gastric microbiota induced by Helicobacter pylori infection and preventive effects of Lactobacillus plantarum ZDY 2013 against such infection. J. Dairy Sci. 99:970-981. https://doi .org/10.3168/jds.2015-10510.
Panwar, H., H. M. Rashmi, V. K. Batish, and S. Grover. 2013. Probiotics as potential biotherapeutics in the management of type 2 diabetes-Prospects and perspectives. Diabetes Metab. Res. Rev. 29:103-112. https://doi.org/10.1002/dmrr.2376.

Phung, O. J., W. L. Baker, V. Tongbram, A. Bhardwaj, and C. I. Coleman. 2012. Oral antidiabetic drugs and regression from prediabetes to normoglycemia: A meta-analysis. Ann. Pharmacother. 46:469-476. https://doi.org/10.1345/aph.1Q554.

Prawitt, J., S. Caron, and B. Staels. 2011. Bile acid metabolism and the pathogenesis of type 2 diabetes. Curr. Diab. Rep. 11:160-166. https://doi.org/10.1007/s11892-011-0187-x.

Qin, J., Y. Li, Z. Cai, S. Li, J. Zhu, F. Zhang, S. Liang, W. Zhang, Y. Guan, D. Shen, Y. Peng, D. Zhang, Z. Jie, W. Wu, Y. Qin, W. Xue, J. Li, L. Han, D. Lu, P. Wu, Y. Dai, X. Sun, Z. Li, A. Tang, S. Zhong, X. Li, W. Chen, R. Xu, M. Wang, Q. Feng, M. Gong, J. Yu, Y. Zhang, M. Zhang, T. Hansen, G. Sanchez, J. Raes, G. Falony, S. Okuda, M. Almeida, E. LeChatelier, P. Renault, N. Pons, J.-M. Batto, Z. Zhang, H. Chen, R. Yang, W. Zheng, S. Li, H. Yang, J. Wang, S. D. Ehrlich, R. Nielsen, O. Pedersen, K. Kristiansen, and J. Wang. 2012. A metagenome-wide association study of gut microbiota in type 2 diabetes. Nature 490:55-60. https://doi .org/10.1038/nature11450.

Rabinovitch, A. 1998. An update on cytokines in the pathogenesis of insulin-dependent diabetes mellitus. Diabetes Metab. Rev. 14:129-151. https://doi.org/10.1002/(SICI)1099-0895(199806)14: 2<129::AID-DMR208>3.0.CO;2-V.

Ren, C., Y. Zhang, W. Cui, G. Lu, Y. Wang, H. Gao, L. Huang, and Z. Mu. 2015. A polysaccharide extract of mulberry leaf ameliorates hepatic glucose metabolism and insulin signaling in rats with type 2 diabetes induced by high fat-diet and streptozotocin. Int. J. Biol. Macromol. 72:951-959. https://doi.org/10.1016/j.ijbiomac .2014.09.060.

Sarkar, D., C. Ankolekar, M. Pinto, and K. Shetty. 2015. Dietary functional benefits of Bartlett and Starkrimson pears for potential management of hyperglycemia, hypertension and ulcer bacteria Helicobacter pylori while supporting beneficial probiotic bacterial response. Food Res. Int. 69:80-90. https://doi.org/10.1016/j .foodres.2014.12.014.

Sheoran, P., and S. K. Tiwari. 2019. Anti-staphylococcal activity of bacteriocins of food isolates Enterococcus hirae LD3 and Lactobacillus plantarum LD4 in pasteurized milk. 3 Biotech. 9:8.

Takemura, N., T. Okubo, and K. Sonoyama. 2010. Lactobacillus plantarum strain No. 14 reduces adipocyte size in mice fed high-fat diet. Exp. Biol. Med. (Maywood) 235:849-856. https://doi.org/10 $.1258 / \mathrm{ebm} .2010 .009377$.

Thomas, C., R. Pellicciari, M. Pruzanski, J. Auwerx, and K. Schoonjans. 2008. Targeting bile-acid signalling for metabolic diseases. Nat. Rev. Drug Discov. 7:678-693. https://doi.org/10.1038/ $\operatorname{nrd} 2619$.

Tian, P., B. Li, C. He, W. Song, A. Hou, S. Tian, X. Meng, K. Li, and Y. Shan. 2016. Antidiabetic (type 2) effects of Lactobacillus G15 and Q14 in rats through regulation of intestinal permeability and microbiota. Food Funct. 7:3789-3797. https://doi.org/10.1039/ C6FO00831C

Toshimitsu, T., J. Mochizuki, S. Ikegami, and H. Itou. 2016. Identification of a Lactobacillus plantarum strain that ameliorates chronic inflammation and metabolic disorders in obese and type 2 diabetic mice. J. Dairy Sci. 99:933-946. https://doi.org/10.3168/jds.2015 $-9916$.

Turnbaugh, P. J., M. Hamady, T. Yatsunenko, B. L. Cantarel, A. Duncan, R. E. Ley, M. L. Sogin, W. J. Jones, B. A. Roe, J. P. Affourtit, M. Egholm, B. Henrissat, A. C. Heath, R. Knight, and J. I. Gordon. 2009. A core gut microbiome in obese and lean twins. Nature 457:480-484. https://doi.org/10.1038/nature07540.

Wang, Q., G. M. Garrity, J. M. Tiedje, and J. R. Cole. 2007. Naive Bayesian classifier for rapid assignment of rRNA sequences into the new bacterial taxonomy. Appl. Environ. Microbiol. 73:5261-5267.

Wang, G., X. Li, J. Zhao, H. Zhang, and W. Chen. 2017. Lactobacillus casei CCFM419 attenuates type 2 diabetes via a gut microbiota dependent mechanism. Food Funct. 8:3155-3164. https://doi.org/ 10.1039/C7FO00593H. 
Xiao, S., N. Fei, X. Pang, J. Shen, L. Wang, B. Zhang, M. Zhang, X. Zhang, C. Zhang, M. Li, L. Sun, Z. Xue, J. Wang, J. Feng, F. Yan, N. Zhao, J. Liu, W. Long, and L. Zhao. 2014. A gut microbiotatargeted dietary intervention for amelioration of chronic inflammation underlying metabolic syndrome. FEMS Microbiol. Ecol. 87:357-367. https://doi.org/10.1111/1574-6941.12228.

Yadav, H., S. Jain, and P. Sinha. 2007. Antidiabetic effect of probiotic dahi containing Lactobacillus acidophilus and Lactobacillus casei in high fructose fed rats. Nutrition 23:62-68. https://doi.org/10 $.1016 /$ j.nut.2006.09.002.

Zhang, F., L. Qiu, X. Xu, Z. Liu, H. Zhan, X. Tao, N. P. Shah, and H. Wei. 2017a. Beneficial effects of probiotic cholesterol-lowering strain of Enterococcus faecium WEFA23 from infants on diet-induced metabolic syndrome in rats. J. Dairy Sci. 100:1618-1628. https://doi.org/10.3168/jds.2016-11870.

Zhang, X., Z. Fang, C. Zhang, H. Xia, Z. Jie, X. Han, Y. Chen, and L. Ji. 2017b. Effects of acarbose on the gut microbiota of prediabetic patients: A randomized, double-blind, controlled crossover trial. Diabetes Ther. 8:293-307. https://doi.org/10.1007/s13300 $-017-0226-y$.
Zhang, X., D. Shen, Z. Fang, Z. Jie, X. Qiu, C. Zhang, Y. Chen, and L. Ji. 2013. Human gut microbiota changes reveal the progression of glucose intolerance. PLoS One 8:e71108. https://doi.org/10.1371/ journal.pone.0071108.

Zhang, Y., X. Guo, J. Guo, Q. He, H. Li, Y. Song, and H. Zhang. 2014. Lactobacillus casei reduces susceptibility to type 2 diabetes via microbiota-mediated body chloride ion influx. Sci. Rep. 4:5654. https://doi.org/10.1038/srep05654.

Zietz, B., A. Schäffler, R. Büttner, J. Schölmerich, and K.-D. Palitzsch. 2000. Elevated levels of leptin and insulin but not of TNF alpha are associated with hypertension in type 2 diabetic males. Exp. Clin. Endocrinol. Diabetes 108:259-264. https://doi.org/10.1055/ s-2000-7998.

\section{ORCIDS}

Nagendra P. Shah ๑ https://orcid.org/0000-0003-4746-3232

Hua Wei ৫ https://orcid.org/0000-0003-4341-8290 